\title{
Human in the Loop Virtual Machine Management on Comet
}

\section{Gregor von Laszewski laszewski@gmail.com}

Fugang Wang, Geoffrey C. Fox, Shawn Strande, Christopher Irving, Trevor Cooper, Dmitry Mishin, Michael L. Norman

\author{
SDCS \& Indiana University
}

Comet is supported by NSF grant: ACI $\# 1341698$ Gateways to Discovery:

Cyberinfrastructure for the Long Tail of Science. 


\section{What is Comet}

- Target the long tail of science

- Focuses primarily on small and modest scale computing jobs,

- Those that require specialized software environments that are not found on traditional clusters.

- Science gateways

- Use Virtual Clusters (VCs) by leveraging existing batch queue, e.g. not OpenStack

- Near-bare metal performing computing resources

- Interactive experience as part of a human-in-the-loop management and usage strategy. 


\section{Hardware}

- $\quad$ 2.76 Pflop/s peak; 48,784 CPU cores; 288 NVIDIA GPUs; 247 TB total memory; 634 TB total flash memory

- Standard Compute Nodes (1944 total)

- Intel Xeon E5-2680v3 2.5 GHz dual socket, 12 cores/socket; 320 GB flash memory; 120 GB/s memory bandwidth

- $\quad$ GPU Nodes (72 total)

- 36 K80 nodes: 2 NVIDIA K80 GPUs per node; dual socket, 12 cores/socket; 128 GB DDR4 DRAM; $120 \mathrm{~GB} / \mathrm{s}$ memory bandwidth; 320 GB flash memory

- 36 P100 nodes: 4 NVIDIA P100 GPUs; dual socket, 14 cores/socket; 128 GB DDR4 DRAM; 150GB/s memory bandwidth; 400 GB flash memory

- Large-memory Nodes (4 total)

- $\quad 1.5$ TB total memory; 4 sockets, 16 cores/socket; $2.2 \mathrm{GHz}$

- Interconnect: Hybrid Fat-Tree topology; $\underline{56 \mathrm{~Gb} / \mathbf{s}}$ (bidirectional) link bandwidth; 1.03-1.97 us MPI latency

- $\quad$ 7.6 PB Lustre-based Parallel File System

- Access to Data Oasis

- High-performance virtualization 


\section{Virtual Clusters on Comet}

- Focus is on giving user a cluster of virtual machines

- Performance is close to bare metal

- Utilizes Infiniband

- Users decide how many of the allocated virtual machines they like to use

- build into accounting and monitoring system of comet 


\section{User Support for Virtual Clusters}

Project

Consultation

Project starts with

consultation to identify

requirements and suitability

\section{Account}

Management

+ Leverages XSEDE accounts and application process,

Users tstart with trial account
Virtual Cluster

Support

( Users have Virtual Cluster support during their $\mathrm{CV}$ activities on Comet

\section{User Support on Comet}




\section{Project Consultation}

- Is the project suitable for VC's on comet?

- Is there enough expertise available <= this has been an issue

- Is there enough time to do the project?

- Are there alternatives that should be used instead?

- Is special support needed? 


\section{Account Management}

- Well defined account management project

- Proven

- Integration with XSEDE

- But

- using of YubiKeys for access to management node

- Integration of user consultation

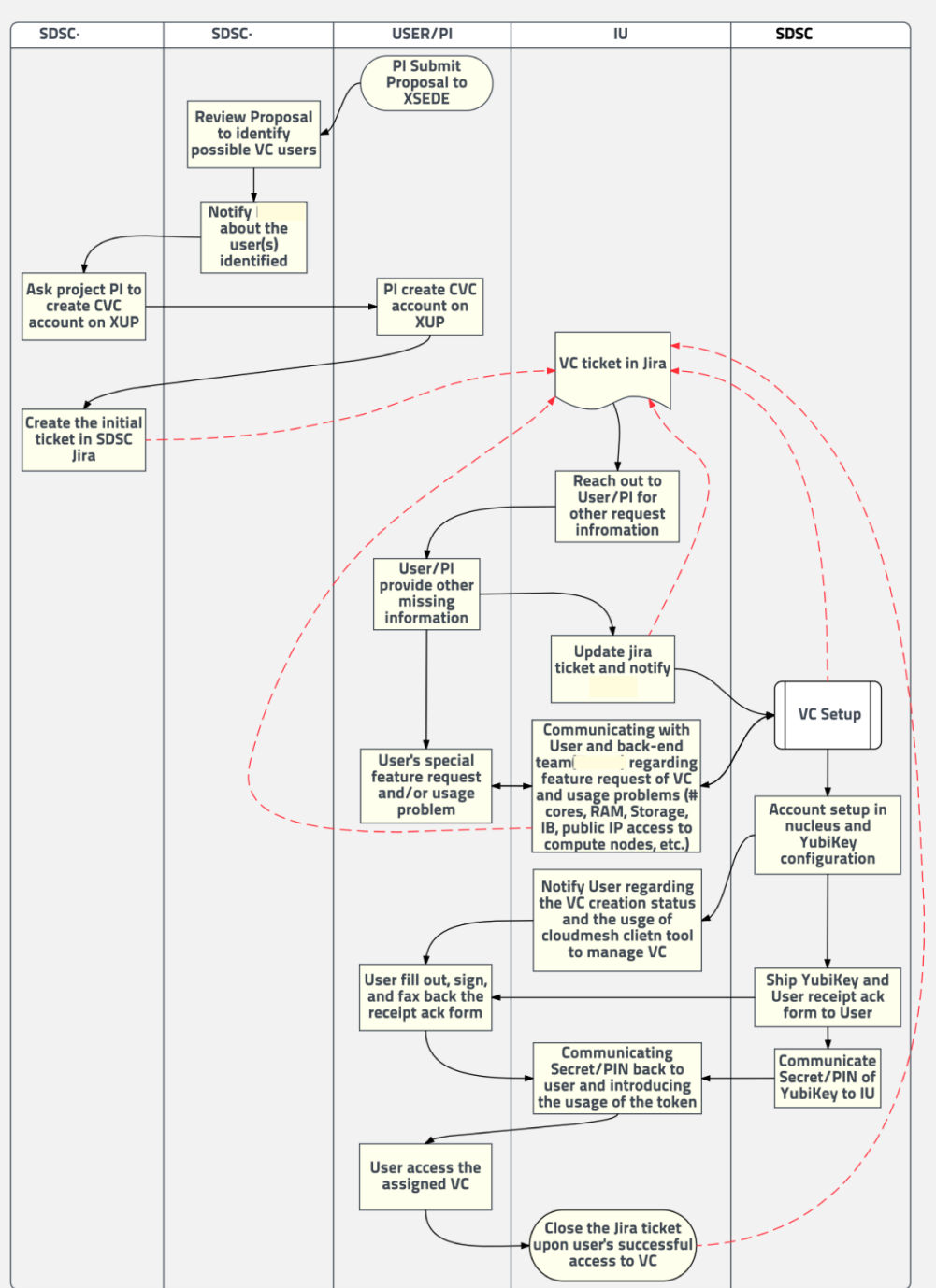




\section{VCs on Comet}

- User gets a virtual cluster

- management through frontend

- Users get $n$ cluster nodes (dependent on need).

- cluster nodes can be user or not

- VCs are all managed by queueing system invisible to the user

- Can use all of the clusters backend services and performance

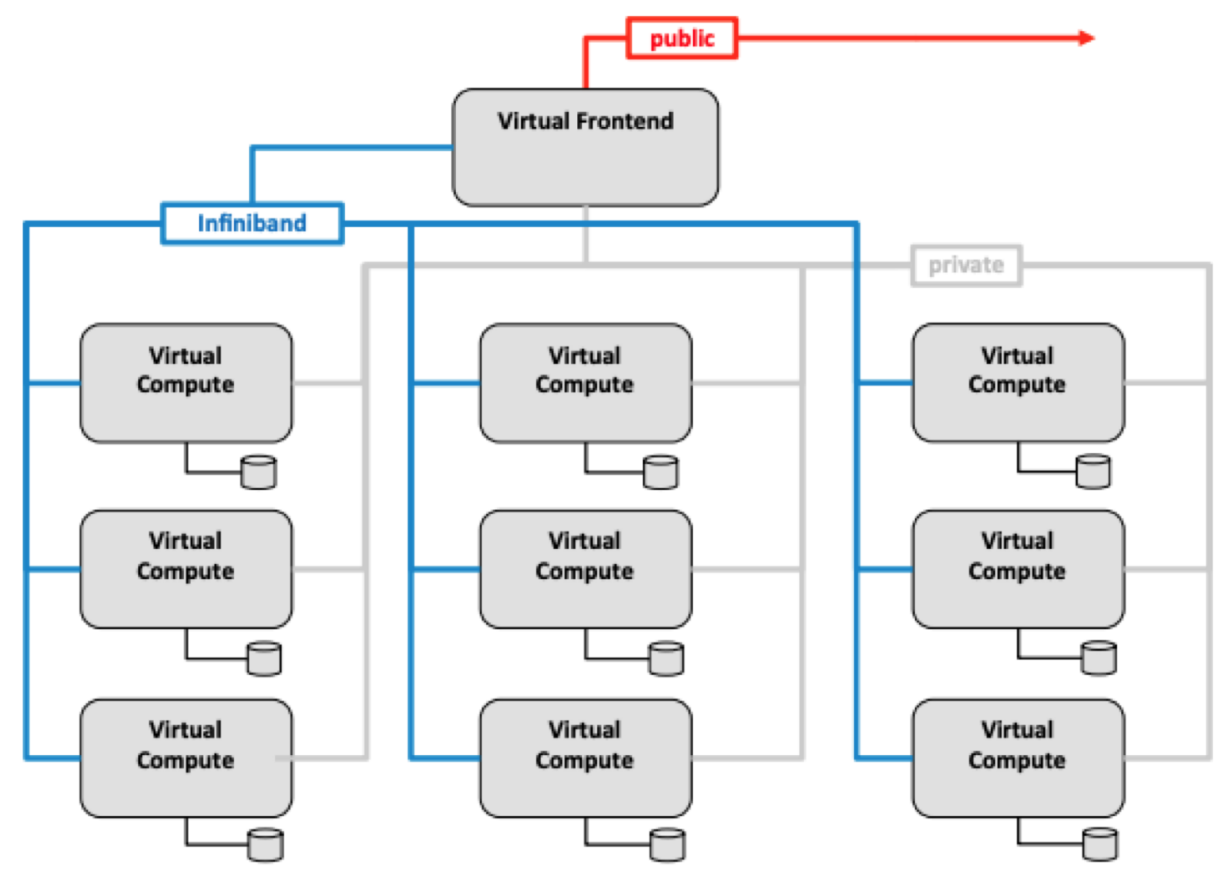




\section{Virtual Cluster Monitoring}

- XSEDE monitoring

- XDMoD integration available

- Special Monitoring

- relatively low wait time
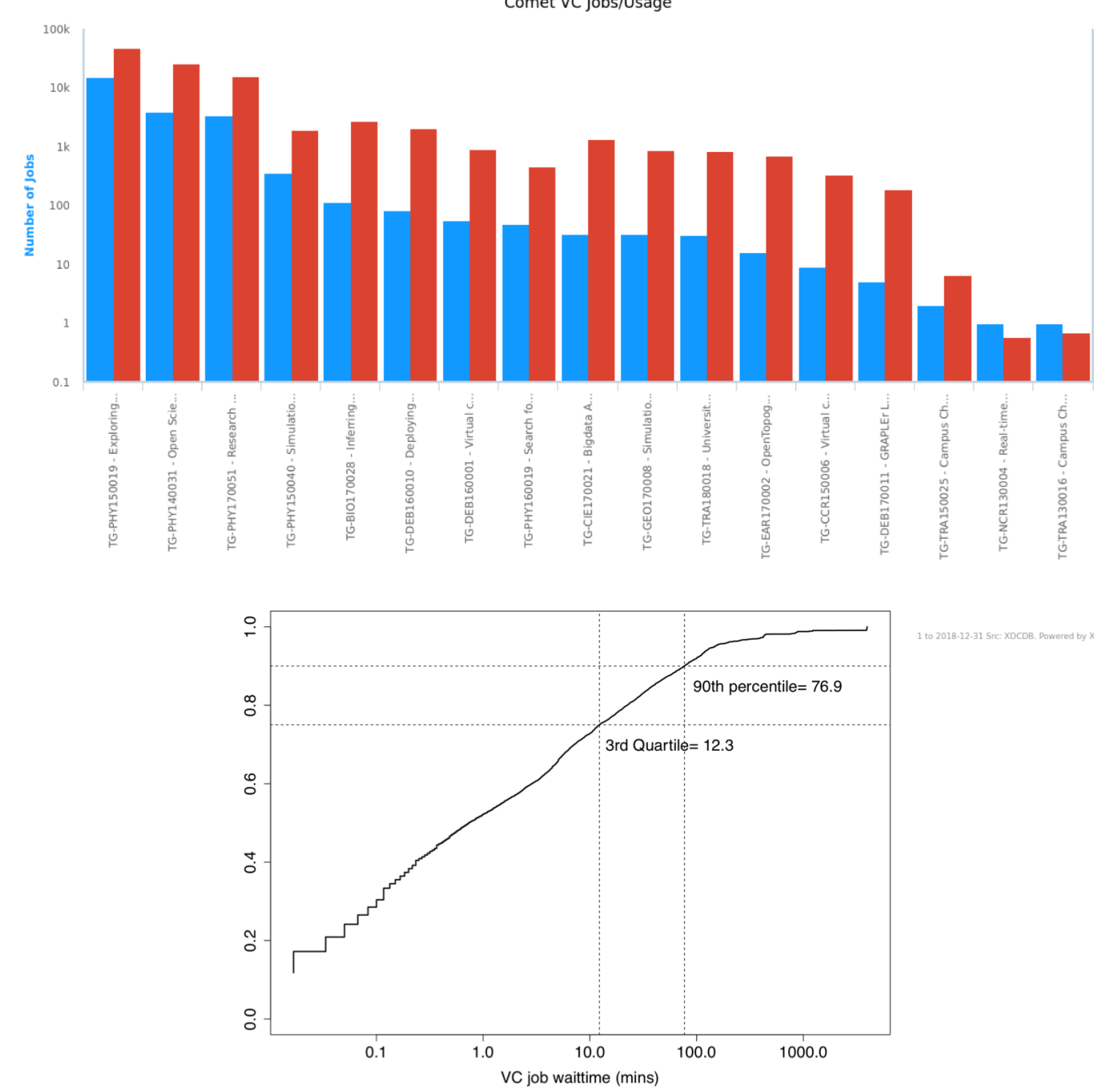


\section{Virtual Cluster Applications}

\begin{tabular}{ll}
\hline Name & Description \\
\hline LIGO & Cluster that integrates in OSG for detection of gravity waves \\
PRAGMA & Virtual Clusters for Environmental Science \\
OSG & Portal to VC Resources \\
Benchmark & Benchmarking petaflop HPC algorithms weather, CFD, and others \\
BigData & VC for evaluation for NIST \\
Darknets & Virtual Clusters for the analysis of darknets \\
THMC & 3D Thermal-Hydrologic-Mechanical-Chemical \\
Performance & tools to capture and analyze memory activities in applications \\
LIDAR & high resolution topography data \\
Biology & Genomic data analysis software stack \\
Astrophysics & HPC-specific workflows for simulating events necessary for precision astrophysical measurements \\
CMS & resources to process 800 Million simulated proton proton collisions in the CMS detector \\
Lifemapper & a high-throughput species distribution (range) modeling system and the main computational platform \\
Education & Campus champion clusters for universities \\
\hline
\end{tabular}




\section{Jupyter Integration}

Cloudmesh can easily be integrated into jupyter

The command shell is readily accessible via an API call

In [4]: $r=\operatorname{shell.cms("help")~}$ In [5]: print (r)

Documented commands (type help <topic>)

$\begin{array}{llllllll}\text { EOF } & \text { container } & \text { help } & \text { key } & \text { q } & \text { shell } & \text { stopwatch } & \text { vm } \\ \text { admin } & \text { data } & \text { image } & \text { man } & \text { quit } & \text { sleep } & \text { sys } & \text { workflow } \\ \text { banner } & \text { default } & \text { info } & \text { open } & \text { register } & \text { source } & \text { var } & \\ \text { clear } & \text { echo } & \text { init } & \text { pause } & \text { sec } & \text { ssh } & \text { vbox } & \\ \text { commands } & \text { flavor } & \text { inventory } & \text { plugin } & \text { service } & \text { start } & \text { vcluster } & \\ \text { config } & \text { group } & \text { ip } & \text { provider } & \text { set } & \text { stop } & \text { version } & \\ \text { Timer: } & 0.0089 \text { (help) } & & & & & \end{array}$

Timer: $0.0089 \mathrm{~s}$ (help)

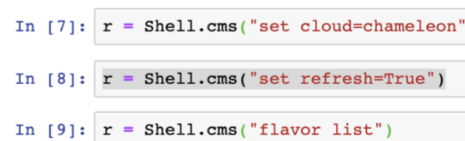

\begin{tabular}{|c|c|c|c|}
\hline Name & | vCPus & RAM & Disk \\
\hline $\mathrm{m} 1$. tiny & 1 & 512 & 1 \\
\hline m1.small & 1 & 2048 & 20 \\
\hline m1.medium & 2 & 4096 & 40 \\
\hline m1.large & 4 & 8192 & 80 \\
\hline $\mathrm{m} 1$.xlarge & 8 & 16384 & 160 \\
\hline storage medium & 1 & 4096 & 2048 \\
\hline m1.xxlarge & ${ }_{16}^{8}$ & $\begin{array}{l}32768 \\
32768\end{array}$ & $\begin{array}{l}160 \\
160\end{array}$ \\
\hline
\end{tabular}


In [1]: from cloudmesh.compute.vm.Provider import Provider

\section{Simple API}

Super simple API that allows integration with jupyter notebooks very easily
In [2]: provider = Provider (name="chameleon")

In [3]: flavors = provider.flavors()

In [4]: flavors [0] [ 'name']

Out [4]: 'ml.tiny'

In [5]: provider.Print(flavors)

\begin{tabular}{|c|c|c|c|}
\hline Name & VCPUS & RAM & Disk \\
\hline------ & & $=-$ & - \\
\hline m1.tiny & 1 & 512 & 1 \\
\hline m1.small & 1 & 2048 & 20 \\
\hline m1.medium & 2 & 4096 & 40 \\
\hline $\mathrm{m} 1$. large & 4 & 8192 & 80 \\
\hline $\mathrm{m} 1 . x$ large & 8 & 16384 & 160 \\
\hline storage.medium & 1 & 4096 & 2048 \\
\hline m1.xxlarge & 8 & 32768 & 160 \\
\hline m1.xxxlarge & 16 & 32768 & 160 \\
\hline
\end{tabular}




\section{Scientific Impact metrics}

- We can alanyls your organizations

- We have done this for scientific impact metrics based on publications

- XSEDE

- We have a unique metric that can compare your peer groups based on publication venues

- NCAR

- Blue Waters

- We could do this based on

- This is different from just i-ndex

- Department

- Research group

- Researcher 


\section{Lessons Learned}

- Advantages: Software

- Superb software, integrates well with existing clusters, including cloudmesh

- Good user support, easy to get access and use a VC

- Backend does not have have "create my comet cluster elsewhere" (not part of funded project)

- Issues: Users

- User knowledge to be a system admin managing the cluster is limited

- Knowledge, manpower, staff retention at organizations or projects

- Issues: Shifting Community Interest

- Community wanted to learn OpenStack

- Shift from VMs to containers 


\section{References}

- Cloudmesh: http://github.org/cloudmesh

- Cloudmesh Comet Plugin: https://cloudmesh.github.io/cloudmesh-comet/

- VC user guide:

- https://cloudmesh.github.io/cloudmesh-comet/comet.htm|\#comet-userguide

- VC CLI reference documentation:

- https://cloudmesh.github.io/cloudmesh-comet/command_comet.html\#comet-command 